\title{
ETNO-SEMIÓTICA DEL RITO: DISCURSO FUNERARIO Y PRÁCTICAS FUNERARIAS EN CEMENTERIOS URBANOS
}

\author{
José Enrique Finol y Karelys Fernández \\ Universidad del Zulia (Maracaibo, Venezuela)
}

«Ritualization is a strategic play

of power, of domination and

resistance, within the

arena of the social body». C. Bell

"C'est ainsi

que la structure des rites

peut servir à emmagasiner

des informations complexes». E. Leach 


\section{INTRODUCCIÓN}

La visita a los cementerios es una práctica extendida planetariamente, y genera en cada sociedad formas particulares de organización que responden a los intereses, ideas y condiciones de cada una de las sociedades y grupos donde tales prácticas se realizan. Para Cannon (1989:437), por ejemplo, «mortuary practice is viewed as a medium for the competitive expression of status and status aspiration». Las prácticas funerarias comprenden un complejo sistema de ritos que abarca desde la preparación del cadáver, el velorio, el entierro y los ritos asociados con el post-entierro. Como comportamiento social constante, de mayor o menor frecuencia según los casos, esas prácticas se han convertido en sistemas rituales ${ }^{1}$ relativamente poco estudiados, en parte porque se diferencian de los ritos oficiales de la iglesia institucional en las modernas sociedades, y en parte porque son expresiones de un proceso sincrético, donde se conjugan influencias de diverso origen que con frecuencia sufren transformaciones propias del lugar, de la clase social y del medio cultural donde las prácticas oficiales establecidas por la iglesia han sido introducidas. En el presente artículo se analizan dos componentes de los rituales que se cumplen durante la visita a los cementerios. Por un lado, se analiza el discurso de los visitantes a los cementerios, en particular se les preguntó sobre el significado que tenía, para cada uno de ellos, la visita periódica a la tumba de un ser querido, familiar o amigo. En la búsqueda de esa información se interrogó a ciento tres personas en dos cementerios

1 En la presente investigación se considera la visita al cementerio como un rito en cuanto constituye un conjunto de acciones que siguen un modelo general y que tienen significaciones específicas en un contexto cultural dado. El rito es, según el punto de vista desarrollado aquí, un sistema semiótico por definición: él expresa valores propios de la sociedad que los practica en un espacio, un tiempo y con actores determinados. Una buena síntesis de lo que el rito comunica la da Monica Wilson: «Rituals reveal values at their deepest level... men express in ritual what moves them most, and since the form of expression is conventionalized and obligatory, it is the values of the group that are revealed. I see in the study of rituals the key to an understanding of the essential constitution of human societies» (Turner, 1969:6). Rappaport es más radical: «I take ritual to be the basic social act» (Bell, 1992:v, 1). En el otro extremo, Staal afirma que: «Man is addicted to ritual activity, a fact that is true of modern society as much as it is true for ancient societies, and that applies to so-called primitive communities as much as it applies to the so-called civilized world» (1982:v), lo cual no le impide luego decir que «Ritual is pure activity, without meaning or goal» (Bell, 1992:v). Honigmann (1953: 6) analiza mejor el punto de vista aquí adoptado: «Ritual we shall define as referring to a patterned configuration of events (including objects, sounds, actions, persons) which (a) embody some necessary or unfailing significance or (b) are appropriate to express the quality felt to be contained in a particular situation». 
ubicados en Maracaibo, ciudad situada en el noroeste de Venezuela, con una población de más de un millón de habitantes, y que constituye un centro petrolero y agropecuario de primera importancia para el país, del cual es la segunda urbe en tamaño después de Caracas. Los cementerios fueron el «Corazón de Jesús», fundado en 1940, y el «San José», fundado en 1925 . Por otro lado, se analizan las prácticas ${ }^{2}$ que los visitantes de los cementerios cumplen durante su estadía en el camposanto. A las mismas personas se les preguntó qué hacían cuando visitaban las tumbas. El propósito era, en un caso y en otro, determinar las recurrencias de las prácticas verbales y comportamentales, con el objeto de elaborar una descripción semiótica de ambos e interpretar el sentido de los mismo en el marco de la cultura funeraria ${ }^{3}$ en Venezuela ${ }^{4}$.

\section{EL DISCURSO FUNERARIO DE LOS VISITANTES DE CEMENTERIOS URBANOS}

\subsection{La visita al cementerio como un acto de comunicación}

Lo mismo que en la sociedad de los vivos, la visita al cementerio constituye un acto de comunicación ${ }^{5}$ que se enmarca dentro de la definición de la cultura como proceso comunicativo (Eco, 1975:42). Para Scarduelli (1988:68), «los ritos representan procesos comunicativos

2 Si bien es cierto que, como afirma Leach (1971:248), «dans les rites, la parole et le comportement sont indissociables», se va a estudiar primero lo que los informantes dicen sobre el significado que otorgan a las visitas a los cementerios y luego el inventario de comportamientos que dicen cumplir en los mismos. La relación entre ambos componentes rituales servirá para mejor interpretar el sentido de los mismos.

3 Se utilizará el término «funerario» no en el sentido restrictivo según el cual se refiere a lo relativo al entierro, sino en un sentido amplio que abarca todas las prácticas que tienen que ver con la relación con los muertos en una cultura determinada. Aun cuando la cultura funeraria en Venezuela está influida en buena parte por la religión católica, ampliamente mayoritaria en el país, las influencias y desarrollo de naturaleza sincrética imprimen a los comportamientos cotidianos relacionados con los muertos características particulares que, como se verá, secularizan lo que originariamente ha podido ser de origen católico.

4 Una caracterización de los visitantes entrevistados fue hecha en Finol y Fernández (1996). Allí se demuestra la presencia dominante de lo femenino en la visita a los cementerios, no sólo de quienes visitan sino también de quienes son visitados.

5 Para un prolijo análisis del rito como un proceso de comunicación véase Fischer (1971) y Finol (1983). 
basados en un código simbólico y (...) los actos rituales constituyen enunciados».

Como se ha señalado en otra parte (Finol y Fernández, 1996), la visita a los difuntos cumple con una estructura similar a la visita social a la morada de los vivos. La repetición de ese esquema de visita evidencia la continuidad de una relación familiar o de amistad que, según la hipótesis aquí presentada, parte del supuesto de la continuidad de la vida después de la muerte, por un lado, y, por el otro, de la presunción según la cual se debe actuar frente a los muertos como si aún estuviesen vivos. Goodman (1988:33) señala el carácter profundo y extraordinario de la comunicación ritual: «The religious ritual, it seems to me, is the most exalted form of human communication, and recognizing this kind of deep structure restores to it that dignity that is lost in the desiccated categorizations of earlier years».

Si se compara la visita a los cementerios con el ritual del velorio, se notará una diferencia fundamental: el velorio es un acto de solidaridad, y por ende de comunicación, con la familia; la visita al cementerio es un acto de comunicación con el muerto. Como ya otros han notado, «death is not a purely individual act, any more than life is. Like every great milestone in life, death is celebrated by a ceremony whose purpose is to express the individual's solidarity with family and community" (Alternatives, 1987:181). Por el contrario, una vez concluida la relación velatoria e incluso el rito del entierro, en los que el individuo y el grupo expresan su solidaridad con la familia, en la relación post-entierro son sólo los familiares quienes quedan solos con su difunto, son ellos quienes, a través de la visita, de las flores y rezos, de la conversación frecuente, conservan viva la relación con los muertos.

De las 144 respuestas que se obtuvieron en las entrevistas a los visitantes a los cementerios, el $13 \%$ señaló que el significado de su presencia frente a la tumba de su familiar o amigo era el deseo de visitarlo. Algunos, por ejemplo, señalaron que venir hasta allí era «como visitar $a$ un ser vivo» ${ }^{6}$. Al vivo se le visita para ver cómo está y al difunto, «para cuidar su lugar de reposo» $(17)^{7}$, para otros, la visita se expresa como el deseo de «estar a su lado un rato» (46), o, más explícitamente, quien expresa que «viene porque le duele su fallecimiento

\footnotetext{
6 Las negritas utilizadas en todas las respuestas citadas son nuestras.

7 Los números entre paréntesis, posteriores a la cita textual de las encuestas, corresponden al número de identificación de éstas.
} 
y quiere hacerle compañía porque está solo en una bóveda» (39) ${ }^{8}$. Asimismo, otros enfocan directamente la visita al objetivo fundamental de la misma: «Para acompañarlo en su soledad»(76). Ahora bien, cuando se examina otro grupo de respuestas, que constituyen el $12 \%$ del total, se observa que bajo otras expresiones lexicales el fondo del discurso constituye el mismo: se trata de vencer la incomunicación y la soledad más allá de la muerte. Para uno de los entrevistados, la visita al cementerio «significa alegría, me gusta venir a compartir con mi hijo (difunto), acompañarlo para que no se sienta solo» (36). Para otro, la visita es «como conversar con ella, visitarla es como hacerle saber que no es olvidada» (80). Un rasgo sorprendente es que en la enorme mayoría de las respuestas dadas no se habla de un contacto, una comunicación o una compañía de orden espiritual; todas las respuestas parecen suponer un contacto que tiene fundamentalmente soportes físicos. Sólo una de las respuestas menciona el orden extraterreno («encontrarme con ellos espiritualmente» (67); los demás parecen suponer que se está hablando siempre de un contacto que, por lo menos, debe afincarse sobre elementos físicos de orden terrenal, elementos que son, como se verá más adelante, los mismos de la vida cotidiana.

Las respuestas examinadas arriba van más allá, al menos para los casos en estudio, de las afirmaciones de Auzías (1976:220), de acuerdo con las cuales «la muerte se halla totalmente escamoteada, mientras que antaño se era responsable del papel que se hacía ante su advenimiento». Las respuestas muestran que frente a la muerte los visitantes imponen un sistema que privilegia la vida, trayendo así a los escenarios de la muerte las mismas prácticas propias de la vida cotidiana.

Si se examina el ritual de la visita funeraria, según lo visto hasta aquí, se verá que por lo menos un $25 \%$ de las respuestas dadas la enfocan como un acto semiótico que busca romper la condena de la soledad que la muerte amenaza con traer a la «vida» de los difuntos. A fin de cuentas, la visita a los difuntos es una duplicación de la visita social que los vivos practican. No se trata de un simple deber o una costumbre - apenas el $4 \%$ de las respuestas menciona esa justificación-, sino, por el contrario, un acto de la pragmática social que hace posible y mantiene el intercambio comunicativo.

8 El entrevistado se refiere al hecho de que, habiendo más espacio para otros cadáveres en la misma bóveda, ésta contenía, hasta ese momento, uno solo. 


\subsection{Semiótica del recuerdo como expresión de la vida: los muertos no se olvidan}

La respuesta dominante en las entrevistas sobre el significado personal de la visita a las tumbas de los deudos es aquella que la vincula con la memoria. En efecto, el $31 \%$ de las respuestas configuran lo que podría denominarse el discurso de la memoria y el recuerdo, el cual aparece opuesto a la soledad y al olvido y se articula en una categoría tímica ${ }^{9}$ donde la memoria y la vida, se suponen y se provocan mutuamente. Véase una muestra de las respuestas que afirman que el significado fundamental del ritual de la visita a los difuntos no es otra cosa que recordar, $\mathrm{y}$, a través del recuerdo, mantener viva la memoria:

— «Tenerlo (al difunto) siempre presente. Hacerle compañía» (4).

- «Sentirse uno bien en visitarle la tumba porque lo recuerdas siempre» (9).

- «Para recordarlos, pues siempre están presentes» (12).

- «No me gusta (visitar la tumba) pero lo hago para no abandonar el recuerdo de mi gente» (25).

- «Vengo a recordar la memoria de mi padre y de mi madre, a traerle flores y a compartir con ellos» (35).

- «Lo visito para recordarlo» (37).

- «Son seres queridos (los padres) que los debemos recordar por siempre, siento que ellos me acompañan en los momentos difíciles» (61).

- «Los recuerdo mucho porque los muertos no se olvidan» (82).

- Como puede observarse, la visita es un acto de recuerdo, un esfuerzo por mantener viva la memoria, para evitar la muerte definitiva. Frente a lo que parece ser un proceso de desemantización de la muerte a través de los medios de difusión masiva, la visita y conservación de la memoria de los muertos es un acto de resistencia cultural que intentaría preservar el valor de la vida como valor fundamental.

9 Ver Greimas y Courtès (1979: 396), quienes establecen que la categoría eufórico/disfórico «juega un papel fundamental en la transformación de los microuniversos semánticos en axiologías: al connotar como eufórica una deixis del cuadrado semiótico, y como disfórica la deixis opuesta, provoca la valorización positiva o negativa de cada uno de los términos de la estructura elemental de la significación». 
Así, la banalización de la violencia y de la muerte en los medios de entretenimiento ha encontrado desde siempre el esfuerzo colectivo por crear mecanismos que conserven el recuerdo y la memoria. Esta hipótesis explicaría el desarrollo ritual de toda una semiótica de la memoria que se fundamentaría en la construcción de procesos comunicativos, pues sólo se mantendría vivo aquello con lo que es posible comunicarse. Aplicado al ritual de la visita a los cementerios, podría esbozarse un esquema semiótico donde la visita es un esfuerzo por mantener la memoria y ésta es una expresión de la continuidad de la vida:

visita $\longrightarrow$ recuerdo $\longrightarrow$ memoria $\longrightarrow$ vida

Para hacer más inteligible esta hipótesis, es conveniente distinguir entre «recuerdo» $\mathrm{y}$ «memoria». La primera connota un proceso, mientras que la segunda connota un estado dado de acumulación de experiencias y conocimientos, de emociones y sensaciones. En este sentido puede decirse que el recordar funciona gracias a la memoria, pero, al mismo tiempo, ésta es mantenida y alimentada por aquél. Si se acepta esta distinción, la visita al cementerio, según los entrevistados, es un proceso recordatorio que permite mantener viva, a través del tiempo, la memoria de los familiares y amigos que han fallecido. Se trataría de una estrategia cuyo objetivo final es impedir que el olvido, como estrategia opuesta al recuerdo, gane terreno a la memoria individual, familiar o colectiva. Correlativamente, puede decirse que, si el recuerdo mantiene la vida, el olvido es, culturalmente, la expresión concreta de la muerte. Es posible representar esa relación con la vieja fórmula binaria de Lévi-Strauss:

recuerdo: vida:: olvido : muerte

donde, : significa «es a....» y :: significa «como».

\subsection{Semiótica del diálogo entre la vida y la muerte}

Como toda relación de auténtica comunicación, la visita al cementerio constituye una relación dialógica donde emisor y receptor interactúan y ambos, de un modo u otro, son afectados por el proceso. Como apunta Curran (1989:450), «...very strong emotional and social 
needs are met through funerary activities». Cuando los entrevistados hablan de su visita y de cómo la interpretan no hablan solamente de una relación unívoca en la que, por ejemplo, el visitante «le hace compañía» al muerto o «le trae flores» o «le reza». En realidad se trata de una relación en dos direcciones. La visita tiene un efecto sobre los vivos y éstos se apresuran a mencionarlo:

— «Significa mucho (la visita), estando aquí me siento bien» (5).

— «Es un alivio» (6).

- «Cumplir con ella y sentirme bien con ella» (7).

- «Sentirse uno bien en visitarle la tumba porque lo recuerdas siempre» (9).

- «Es lo más grande del mundo (la visita), tiene tres años de muerto y me siento feliz de irla a visitar» (10).

- «La recuerdo mucho, siente uno satisfacción con el hecho de visitarlo» (11).

— «iento consuelo de ser lo único que puedo hacer» (14).

- «Hallo consuelo visitándolos (a sus padres). Cuando me siento triste visito la tumba y me siento mejon» (26).

Para muchos otros, la visita al cementerio permite conseguir «un consuelo» o les proporciona «regocijo» o simplemente es «reconfortante». Para otros, «son seres queridos que los debemos recordar por siempre. Siento que ellos me acompañan en los momentos difíciles» (61). Asimismo, otros declaran: «Vengo aquí para sentirme bien, para desahogarme con el difunto» (79). Pero quizás quien mejor expresa el sentido profundo de la visita es aquel que reconoce que ello le proporciona «una paz interna porque sabe que lo ha visitado». Este discurso evidenciaría que no sólo se visita a los muertos por el bien de éstos, para que no se sientan solos o para que las oraciones les traigan descanso eterno o paz a sus restos; se les visita también para que traigan paz, consuelo y bienestar a quienes aún continúan vivos. Un tema recurrente en la conversación con los muertos (el $30 \%$ de los entrevistados dice que cuando va al cementerio conversa con el difunto) es pedirles «que intercedan por los que estamos todavía vivos». Así pues, el discurso funerario, la comunicación entre vivos y muertos, es un diálogo, una conversación directa, un contacto presencial que se distingue claramente de la comunicación a través del ritual oficial de la iglesia. Cuando los deudos acuden a las 
misas de cumplemés o de aniversario ${ }^{10}$ tienen con el difunto una relación que podría llamarse vicaria, pues es a través del sacerdote como la comunicación se establece con Dios, con el propósito de que éste, finalmente, conceda paz y dicha eterna a la persona fallecida. La comunicación con el difunto, en el cementerio, es directa, no requiere de intermediarios y, más importante aún, se trata de una comunicación en presencia, en cuanto que ésta se desarrolla en el lugar donde los restos físicos reposan. Mientras que durante el novenario o durante las misas de cumplemés y aniversario el difunto está ausente físicamente, en la visita al cementerio éste está presente físicamente, aunque no sea visible; mientras que en la misa no hay ningún recordatorio físico de la persona fallecida, en el cementerio, en su tumba, hay una lápida con su nombre, su fecha de nacimiento y muerte. Por esta vía es fácil, como se verá, asimilar la tumba al hogar, mientras que la iglesia, en cuanto espacio, es difícilmente asimilable al hogar: mientras que en este último la comunicación es directa, como lo es en el cementerio, en la iglesia la comunicación es a través de la mediación del sacerdote y en todo caso no tiene como destinatario al propio difunto sino a la divinidad.

Basándose en la argumentación precedente, es posible proponer una hipótesis que haga más inteligible el sistema semiótico desarrollado hoy alrededor del ritual de la visita al cementerio. Si como se ha dicho, los rituales oficiales que tienen lugar en la iglesia en cierto modo excluyen la presencia del difunto, pues se trata de una relación entre deudos, sacerdote y divinidad que, en todo caso, es sólo a propósito del difunto, podría argumentarse que tales rituales suponen que el difunto está en verdad y definitivamente muerto, mientras que en el ritual de la visita al cementerio el visitante, envuelto en una relación personal y directa con la presencia física del difunto, actúa como si el difunto aún estuviera vivo. Si esta hipótesis es correcta, la pregunta inmediata es por qué el visitante actúa así. Antes de responder, deben reunirse otros elementos que den fuerza heurística a la

10 Usualmente, en la tradición funeraria católica que se practica en Venezuela, después de fallecida la persona se realizan nueve noches continuas de rosario; este rito es conocido como Novenario y su objetivo es lograr el descanso eterno, en el Paraíso, de la persona fallecida. Anteriormente, estos rosarios se realizaban en la misma casa del difunto. Hoy han sido progresivamente sustituidos por nueve misas $o$, en ocasiones, el Novenario se practica en alguna iglesia local. Algunas familias acostumbran luego ordenar una misa en cada cumplemés durante el primer año posterior a la muerte. De allí en adelante se acostumbra hacer una misa cada año en la fecha aniversaria del fallecimiento. 
respuesta a elaborarse. Conviene analizar ahora las conductas rituales que los visitantes dicen cumplir cuando visitan las tumbas en los cementerios.

\section{PRÁCTICAS FUNERARIAS EN CEMENTERIOS URBANOS}

\subsection{En el cementerio como en la casa}

Si, como afirma Greimas (1979b:12), «l'homme est le signifié de tous les langages», el lenguaje espacial propio del cementerio y de las tumbas hace que éstas no sólo sean lugar sino también espacio, es decir, una coordenada física llena del sentido humano que le otorga la presencia de los visitantes en diálogo constante con los visitados. En otros términos, en ese lugar tan particular de la ciudad el hombre ha elaborado una semiótica topológica, es decir, le ha dado un sentido que, como se verá, es homologable en varios aspectos con el espacio que se conoce como hogar o morada familiar. No se trata, pues, de lugares vacíos de sentido, sino que la presencia del ser humano y las prácticas que éste cumple han introducido discontinuidades espaciales, no sólo significantes sino también significativas.

Villa Posse (1993:91) afirma que «en mausoleos, tumbas y aún en el diseño las lápidas buscan en sus formas arquitectónicas la simulación de casas o templos». Esta identificación entre tumbas y casas, también puesta de manifiesto por Thomas (1983), se confirma cuando se examinan las conductas que los visitantes dicen cumplir cuando van al cementerio.

Según Vovelle y Bertrand (1983:102) en su investigación sobre el «imaginario urbano contemporáneo según los cementerios de provincia», durante la visita a las tumbas se cumplen tres «gestos» principales: a) un breve momento de recogimiento (oración, meditación?), b) colocación de flores frescas, y c) limpieza de la tumba. En la presente investigación, la conducta más frecuente, no reseñada por Vovelle y Bertrand, es la de conversar con el difunto (30\%). Más de la mitad de los entrevistados que dicen conversar con sus deudos afirman que le cuentan «los acontecimientos familiares». Esta conducta pone en evidencia dos aspectos significativos de la relación vivos-muertos, tal como se la construye en estos sencillos rituales cotidianos. Por un 
lado, la conversación como tal (éste es el término utilizado por la mayoría) muestra una vez más un esfuerzo por reparar la discontinuidad de la relación creada por la muerte física. Conversar, el proceso por antonomasia que hace posible la comunicación, permite oponerse exitosamente a la separación física que produce la muerte y permite, asimismo, hacer que la memoria se actualice gracias al recuerdo. Por otro lado, la práctica de contar al difunto los últimos acontecimientos familiares busca mantener ya no sólo la continuidad entre el difunto y el individuo, sino también entre el primero y la familia como un todo. Ahora bien, así como la casa es escenario privilegiado de la conversación y asiento natural de la familia, también el cementerio lo es. El cementerio es lugar de conversación, como ya se ha visto, y es lugar donde los familiares se reúnen con sus familiares. Es lógico así homologar la tumba a la casa: ambos son escenarios de comunicación y diálogo, ambos son encuentro del núcleo familiar, ambos son lugar de reunión y descanso. En 1979 uno de los encargados de mantenimiento en el cementerio de San Pierre, en Marsella (Francia), señalaba que, en efecto, tumba y casa son comparables. Al hablar de la primera, decía que ésta «c'est une maison, même qu'on l'habite pas tout le temps, on y viendra bien tôt ou tard finalement» (Vovelle y Bertrand, 1983:103) (subrayado de los autores). En otros términos, el sistema semiótico que se genera y se transforma en la casa o lar familiar es en no pocos aspectos el mismo que se crea alrededor de la tumba.

\subsection{La limpieza de la casa}

Otra de las conductas más practicadas en los cementerios por quienes visitan a sus deudos es la de limpiar las tumbas. En los cementerios donde se realizaron las encuestas, esta limpieza es una tarea relativamente fuerte, pues se trata de mausoleos grandes y no de simples lápidas colocadas sobre la tierra. Siguiendo la tradición funeraria monumental española y católica, los dos cementerios donde se realizó la pesquisa están constituidos en su gran mayoría por monumentos y bóvedas que ocupan espacios tan grandes, por lo menos, como el tamaño del sarcófago que se sepulta. Ahora bien, la limpieza de las tumbas es sentida por los deudos como una responsabilidad ineludible. Algunos sepultureros informan que varias familias pagan regularmente a los responsables del mantenimiento de los camposantos, a fin de que 
mantengan las tumbas de sus deudos limpias de tierra y arbustos. El empeño por la limpieza de las tumbas es comparable con el empeño por la limpieza de la propia casa. Vovelle y Bertrand (1983:102) agregan que en los cementerios franceses de provincia ésta es una tarea que generalmente realizan las mujeres, y hay en ella una marcada división de clases: las clases populares limpian ellas mismas las tumbas, mientras que las clases económicamente mejor situadas contratan empresas que se encargan de esa tarea, así como de la colocación de flores.

En el medio aquí analizado, mientras que ni el individuo ni la familia sienten obligación por la limpieza de lo que son espacios públicos (la plaza, la calle, etc.), en el cementerio, a pesar de ser un espacio público, los deudos de los familiares conciben el espacio de la tumba como propio y por lo tanto se sienten obligados a limpiarla y a mantenerla. Una primera explicación fácil sería que las tumbas, en efecto, son propiedad privada: fueron adquiridas y pagadas por la familia" ${ }^{11}$ En tanto tal, no son públicas sino de propiedad privada, y ello explicaría el interés por mantenerlas limpias. Esa respuesta no parece suficientemente satisfactoria. La verdadera razón por la cual ese espacio mortuorio es sentido como propio, y en consecuencia como espacio que debe ser mantenido y cuidado, es que allí yace un miembro de la familia, es parte de la misma, y en tal sentido es una prolongación del lar familiar ${ }^{12}$. Esta hipótesis se alimenta del hecho ya señalado, según el cual las mismas conductas que ocurren en la casa o residencia, ocurren también en el cementerio ${ }^{13}$.

Si se analiza a nivel profundo la inserción del valor «limpieza» en el sistema semiótico que se ha venido esbozando desde el inicio, se verá que así como las estrategias comunicativas con los difuntos buscan mantener viva la memoria y, en consecuencia, la vida, igualmente la estrategia ritual de la limpieza está estrechamente vinculada con la negación de la muerte o, en otros términos, con la recuperación de la vida. La muerte, concebida como descomposición y destrucción, como desaparición y vacío, es contrarrestada a través de estrategias rituales (conversación, memoria, limpieza) que permiten a la sociedad afirmar el valor de la vida por encima de la muerte definitiva y total. Un espacio limpio y

\footnotetext{
11 Para Vovelle y Bertrand (1983:102) «le nettoyage peut aussi etre un geste symbolique de réaffirmation des droits d'une famille sur un lopin du cimetière».

12 Vovelle y Bertrand (1983:102) afirman que durante la limpieza la tumba se convierte en «le double symbolique de l'être aimé».

13 «La tumba es lugar de "reposo" o de "descanso", actividades que habitualmente, en el mundo de los vivos, se cumplen en el hogar, de allí la homologación constante, en la creencia popular, entre el hogar y la tumba» (Finol y Fernández, 1996).
} 
mantenido es un espacio que cierra el paso a los avances del olvido que es percibido como la muerte definitiva.

\subsection{Flores y velones: belleza y luz}

En la estrategia ritual arriba mencionada cumplen un papel determinante otras prácticas que los entrevistados señalan. Se trata del aporte de dos objetos tradicionales en la cultura funeraria cristiana. En primer lugar está el uso de flores y en segundo lugar, el de velas o velones. Un $30 \%$ de los encuestados señaló que trae flores o velones a los difuntos. Ambos objetos, utilizados para adornar las tumbas, tienen el propósito de expresar vida. Las flores son fruto de la vida, tienen como objeto embellecer lo que de otro modo sería visto como feo e irrecuperable, y a través de sus colores vivos buscarían contrarrestar el tradicional color negro asociado con la muerte. El uso de las flores se percibe como una articulación sémica que se opondría cromáticamente a los valores asociados con la muerte en el código funerario católico. El uso de velas y velones que son encendidos sobre las tumbas es visto también como parte de la construcción de un sistema cultural que afirma la vida por encima de la muerte. La luz de las velas estaría así contrapuesta a la oscuridad de la muerte. Si bien el fuego tiene innumerables significaciones, una de las principales es aquella que se deriva de su oposición a la oscuridad. En la simbología cristiana tradicional la luz es sinónimo de vida y de purificación.

Durante las visitas realizadas a los cementerios es notable la progresiva sustitución de las flores naturales por las artificiales, lo que no sólo refleja un proceso ya largo en la cultura occidental que se ha llenado de numerosos objetos plásticos, los cuales tienen sobre los objetos naturales la posibilidad de una mayor duración frente a la acción destructora del tiempo. Esto es particularmente válido en el caso de las flores, cuya durabilidad es tan corta una vez que han sido separadas de la planta donde crecieron. La dialéctica semiótica que se origina en el enfrentamiento entre lo natural y lo artificial facilita la comprensión de un nuevo significado de la presencia de las flores diferente de la ornamentalidad antes reseñada. En efecto, como apuntan Vovelle y Bertrand (1983:102), las ofrendas florales, lo mismo que la limpieza de las tumbas, «constituent des preuves visibles que la tombe a été visitée». Urbain (1978:435) apunta igualmente que 
«une des fonctions fondamentales des fleurs sur les tombeaux est d'être le signe, lorsqu'elles sont fraîches, de fréquentes visites». Las flores devienen así, más allá de su valor ornamental, signos que buscan comunicar, tanto a los demás visitantes al cementerio como a otros familiares que pudiesen venir en otro momento, que la tumba ha sido cuidada y visitada, que el muerto ha sido recordado. Como en otros rituales, la visita al cementerio es un proceso semiótico que involucra, en tanto receptores, no sólo a los actores participantes en el mismo proceso sino también a los espectadores miembros del grupo o de la comunidad.

\subsection{El rezo}

La cuarta actividad más practicada por los visitantes entrevistados, después de la conversación, la colocación de flores y velones y la limpieza de las tumbas, es el rezo. La práctica de la oración es lo que define mejor el carácter religioso de la visita funeraria a pesar de que sólo el $19 \%$ la mencionó. Ello parecería indicar, si se toma en cuenta la relativa escasez de esta respuesta, una progresiva secularización o, mejor, una pérdida del carácter religioso de la visita al cementerio. En lugar de rezar los visitantes entrevistados preferirían conversar con sus difuntos (30\%), traer velones y flores (30\%) o limpiar las tumbas (18\%). La oración por los difuntos aparecería, comparada con las otras tres acciones más frecuentes señaladas, como una expresión o afirmación de la muerte: se reza para buscar de la divinidad un premio o recompensa eterna para el deudo fallecido, para quien ha muerto. Los otros tres comportamientos reseñados apuntan, individualmente y en su conjunto, hacia una expresión más cercana a la vida, a las conductas y valores de la vida como definición inicial y fundadora de la cultura humana.

\section{EL SISTEMA SEMIÓTICO: VIDA Y MUERTE}

Se debe ahora reorganizar los contenidos dispersos mencionados a lo largo del análisis, con el propósito de elaborar un modelo que sea coherente y que permita aproximarse a la semiosis ritual que se 
cumple a diario en los cementerios estudiados. Como en todo ritual funerario, las conductas de los participantes están orientadas a construir formas de relacionarse con la muerte, con su indefinible y definitiva recurrencia, con la incapacidad para dominarla y controlarla. Como en todo ritual, también aquí se trata de ordenar el caos, de reducir el azar y limitar la angustia para entonces hacer posible la vida. Es en torno a esos dos extremos de la condición humana, muerte y vida, como el ritual funerario se inserta, se organiza, desarrolla sus estrategias y trata de resistir, oponerse y ganar control. En el gráfico siguiente se esquematizan expresiones de dos valores de la semiótica fundamental del ritual:

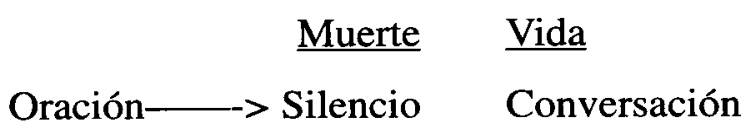

Olvido $\quad$ Recuerdo $\longrightarrow$ Memoria

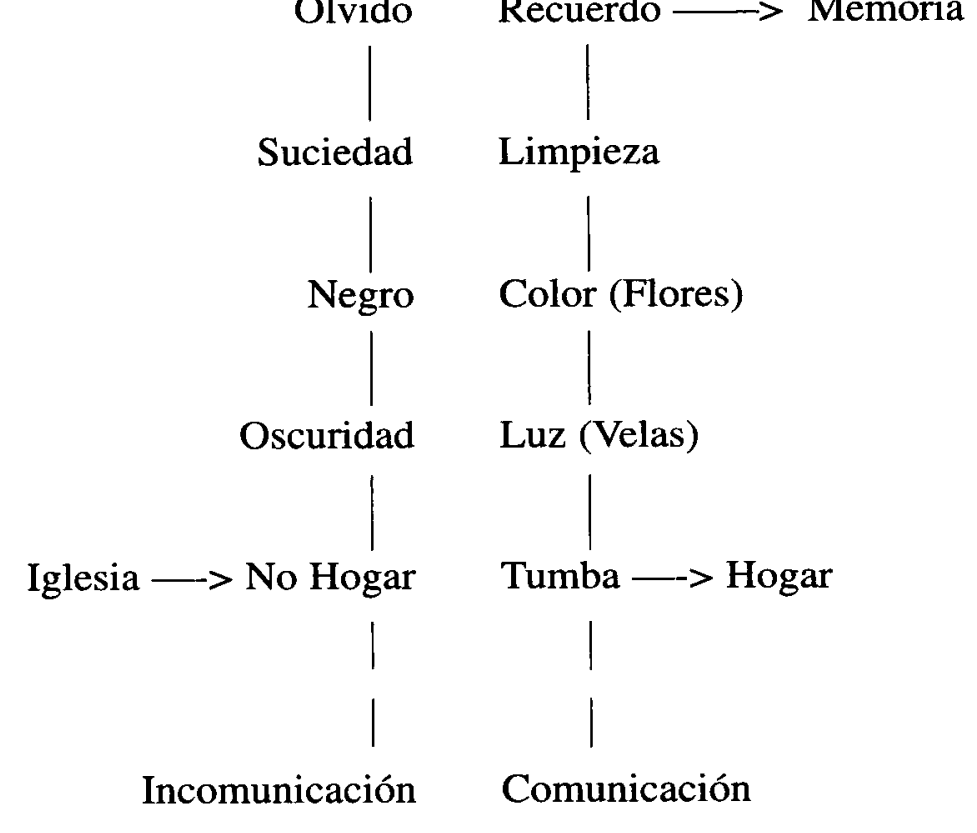

Este esquema, a pesar de su rigidez y simplicidad, muestra cuáles son los elementos y cuáles, las estrategias utilizadas por los participantes en este ritual funerario que se realiza particularmente los fines de semana, el Día de los Difuntos y en las fechas aniversarias, 
cuando familiares y amigos ${ }^{14}$ asisten a los cementerios a visitar «las moradas de nuestros muertos». Allí, en silenciosas comunicaciones y en sencillas prácticas de conversación, limpieza, oración, adorno y alumbramiento, los vivos tratan de mostrar que la vida es superior a la muerte. Son todas esas interacciones verbales y rituales las que permiten desarrollar la semiosfera ${ }^{15}$ gracias a la cual el sistema cultural afirma que es posible no sólo vivir sino triunfar sobre la muerte.

\section{CONCLUSIONES}

El análisis precedente ha mostrado elementos fundamentales que articulan el sistema semiótico sobre el cual descansan las prácticas discursivas y comportamentales propias de lo que se ha denominado el ritual de la visita a los cementerios. El conjunto de elementos encontrados esboza un modelo que rige esos comportamientos y permite acercarse a una mejor comprensión de cómo los grupos entrevistados perciben, no importa si consciente o inconscientemente, las relaciones entre la vida y la muerte, entre la sociedad de los vivos y la sociedad de los muertos; permite también formular con propiedad hipótesis que ayuden a comprender cómo el sistema social y el sistema cultural enfrentan situaciones que están más allá de su control y que en consecuencia crean incertidumbre y angustia. Frente a ello parecería necesario introducir estrategias culturales que permitan vivir a pesar de la angustia de la muerte ${ }^{16}$.

14 Con una sola excepción, todos los encuestados dijeron estar visitando no amigos sino familiares muertos, de los cuales el $91.79 \%$ estaba vinculado al visitante por nexos de consanguinidad y el $8.21 \%$, por nexos de afinidad, lo que prueba que la visita es fundamentalmente a un familiar fallecido. Se traraía, pues, de un acto familiar. El 78.63\% de los visitantes encuestados en el cementerio era de sexo femenino, mientras que una proporción casi exactamente similar, el 76.65\%, de los difuntos visitados era también de sexo femenino (Finol y Fernández, 1996).

15 Lotman (1990:123-24) define la semiosfera como «the semiotic space necessary for the existence and functioning of languages, not the sum total of different languages; in a sense the semiosphere has a prior existence and is in constant interaction with languages». Lotman agrega que «outside the semiosphere there can be neither communication, nor language».

16 Miceli (1975:153-54) ha expresado con precisión cómo el rito actúa de forma determinante en el control de la angustia: «Il rito in altre parole ha effettivamente la capacità (e non c'è religione che non lo sappia come non c'è ideologia che non finisca per apprenderlo) d'imporre, sulla la contingente imprevedibilità degli eventi, la 
Es necesario ahora que se responda la pregunta planteada en 2.3. En efecto, si es correcta la hipótesis según la cual en realidad los visitantes entrevistados actúan como si el difunto estuviese vivo, habría que preguntarse por qué y, en consecuencia, cuál es la ideología que actúa detrás de ese comportamiento. Como se ha sostenido antes (Finol y Fernández, 1996), el ritual funerario no busca, como sostiene Thomas (1983), circunscribir o entrampar la muerte. El ritual que hemos analizado busca negar la muerte porque de esa manera afirma la vida y, afirmando la vida, toma el control sobre la circunstancia adversa, sobre la angustia, sobre el azar y la nada. Es analizando lo que Bradley (1989:448) llama «strategies of the living», como es posible formular una hipótesis que haga inteligible la relación que los visitantes del cementerio establecen con sus muertos. La estrategia comunicativa que rige todo el culto funerario estudiado aquí, recurre a todos los elementos propios de la vida - la conversación, la limpieza, las flores, la luz, la extensión de la morada familiar - porque al final el actor quiere identificarse con la vida, que es el componente esencial de la cultura. Cuando uno de los informantes señalaba que para ella sus seres queridos «nunca han muerto» o, como afirmaba otro, que le agradaba «venir a visitar a sus padres a este lugar (al cementerio) que al final será la casa de uno», se está afirmando que es necesario mantener la continuidad de la vida porque es la vida la que cuenta, la que existe, la que domina ${ }^{17}$. Al afirmar la vida, se está haciendo un acto de resistencia $^{18}$ que busca colocar al ser viviente en posición dominante, que trata de alejarlo de su condición de víctima de la muerte; se trata, finalmente, de una estrategia destinada a tomar control. Como se ve, el ritual vuelve a ser, como sugiere Bell (1992), una estrategia de poder, de adquisición del control ${ }^{19}$. Como acto de resistencia frente a situa-

dimensione della certezza, de la necessità e dei valori assoluti. (...) É infatti la forma strutturale del rito che, essa stessa, si costituisce come una vera e propia sfida alla contingenza e alla imprevedibilità dei procesi della storia violando, e anzi puntualmente invertendo, le regole normali secondo le quali qualunque evento è obbligato a manifestarsi».

17 En la dialéctica entre la vida y la muerte los cementerios son objeto de esfuerzos por conservarlos como objetos y espacios con signos de vida. «Cemeteries are caught in the intersection of reverence and rebellion; the demands of grief and memory co-exist with our need to affirm life, against which cemeteries stand in opposition" (Voller, 1991: 8). Es en un esfuerzo por doblegar esa oposición como la «sociedad de los vivos» lucha para reducir el poder del olvido y de la muerte.

18 Para un extenso análisis del rito contemporáneo como forma de resistencia, véase Hall y Jefferson (1976).

19 «Ritualization is first and foremost a strategy for the construction of certain types of power relationships effective within particular social organizations» (Bell, 1992: 197). 
ciones incontrolables, el rito posibilita construir un nuevo equilibrio que dé sentido a la condición del ser humano como ser viviente: «Di fronte a situazioni altrimenti incontrollabili i rituali, che prescrivono precisi modi d'agire, sappiano dare sicurezza e fiducia o che essi, fornendo mezzi istituzionalizzati per incanalare emozioni e sentimenti, abbiano una efficacia equilibratrice» (Miceli, 1975:132). Curran (1989:450), agrega que «death is (...) a threat to the existing family order», lo que explica el esfuerzo, a través de diversas estrategias rituales, para enfrentar de modo sistemático la amenaza que la muerte representa para el orden familiar de la vida y para su necesidad de continuar la existencia.

Ahora bien, la estrategia ritual fundamental contra la muerte es el recuerdo, es la lucha contra el olvido. Si, como afirma Thomas, «la mort est un silence», los vivos se oponen a ella a través de signos que comuniquen la presencia de la vida, creando así una semiótica funeraria que cree la ilusión de que la muerte, al fin de cuentas, también puede ser derrotada. «Ces mots, ces signes, ces images, ces symboles son là pour faire du bruit au coeur du silence de la mort: ils son là pour y introduire un parasitage signifiant, donc rassurant!» (Thomas, 1978:9). Es exactamente esa misma imagen de vida lo que también busca crear el director funerario cuando prepara el cadáver antes de ser expuesto en el ataúd. Allí, de acuerdo con Barley (1983), se trata de crear, a través de lo que él denomina «códigos de restauración», el sentido de naturalidad y normalidad. Esos códigos son los de pose, cosmético, vestimenta y posición, los cuales facilitarán la creación de un sentido contrario al que la cultura funeraria le atribuye a la muerte. También la construcción de una semiótica de la vida parece estar presente en los propios textos de las lápidas funerarias: "Remembering» life is a primary function of the funerary text, even if the reader of that text is a stranger" (Voller, 1991:8).

Nada se parece más a la muerte que el olvido; de ahí el esfuerzo denodado que hacen los grupos humanos por evitar que el olvido gane terreno a la memoria. Una de las formas que el olvido ha tomado en cementerios europeos es el anonimato. Urbain, en su visita a más de 250 cementerios europeos, ha encontrado en Holanda algunos ejemplos de una naciente desaparición de los nombres propios en las tumbas, los cuales han sido sustituidos por sustantivos generales: «Hier rust PAPPA. 1899-1959, en Mamma. 1899-1972» (Aquí descansa PAPA 1899-1959 y MAMA 1899-1972) (Urbain, 1978:449). En otros, simplemente, se encuentra «Tumba del Padre» o «Tumba de la 
Madre». Otro proceso que evidenciaría el comienzo de la desaparición de la Société de Conservation, siempre según Urbain, sería el progreso de la incineración. Estos fenómenos, no obstante, no se han detectado en los cementerios visitados en Venezuela, donde la lucha por la conservación de la memoria de los muertos, a pesar de la evidente reducción en el número de visitas a los cementerios, aún continúa.

Un estudio sincrónico como el presentado aquí debe ser completado con un estudio diacrónico que permita ver las variaciones ${ }^{20}$ a través del tiempo, en el ritual estudiado. Un intento por establecer relaciones entre los cambios culturales, sociales y económicos y las prácticas rituales en general, las funerarias en particular, contribuirá decisivamente a la comprensión de la cultura funeraria y de la concepción de la muerte que ésta incluye. Sólo así se tendrá una idea más clara de la evolución de la ideología que subyace en las relaciones necesariamente cambiantes entre los muertos y los vivos.

\section{Referencias bibliográficas}

Alternatives (1987). To Celebrate. Reshaping Holidays and Rites of Passage. Ellenwood (Georgia): Alternatives.

AUZIAS, J.-M. (1976). La Antropología Contemporánea. Caracas: Monte Ávila. BARLEY, S. (1983). «The Codes of the Dead: the semiotic of funeral work». Urban Life 12, 3-31.

Bell, C. (1992). Ritual Theory Ritual Practice. New York-Oxford: Oxford University Press.

Bradley, R. (1989). Comentarios a Cannon, A.: «The Historical Dimension in Mortuary Expressions of Status and Sentiment». Current Anthropology $30: 4,448-449$.

CANnon, A. (1989). «The Historical Dimension in Mortuary Expressions of Status and Sentiment». Current Anthropology 30:4, 437-458.

Chapman, R.W. (1989). Comentarios a Cannon, A.: «The Historical Dimension in Mortuary Expressions of Status and sentiment». Current Anthropology 30:4, 449.

Curran, M.L. (1989). Comentarios a Cannon, A.: «The Historical Dimension in Mortuary Expressions of Status and Sentiment». Current Anthropology 30:4, 449-450.

20 Chapman señala que ya van Gennep y Hertz «viewed variation in such practices (rites of passage) as the result of relationships between the corpse, the soul, and the survivors» (Chapman, 1989:449). 
Eco, U. (1975). Trattato di Semiotica Generale. Milano: Bompiani.

FINOL, J. E. (1983). Semiótica, Comunicación y Cultura. Maracaibo: Heurískein.

FINOL, J. E. y FERNÁNDEZ, K. (1996). «Socio-Semiótica del Rito: Predominio de lo Femenino en Rituales Funerarios en Cementerios Urbanos». Manuscrito.

FISCHER, E.A. (1971). «Ritual as communication». Worship 45, 73-91.

Goodman, F. (1988). Ectasy, Ritual, and Alternate Reality. Bloomington: Indiana University Press.

Greimas, A. J. (1979). «Pour une sémiotique topologique». En Sémiotique de l'Espace, 11-43. París: Denoel/Gonthier.

Greimas, A. J. y Courtès, J. (1979). Sémiotique. Dictionnaire Raisonné de la Théorie du Langage. París: Hachette.

HALl, S. y JeFFERSON, T. (ed.)(1976). Resistance through Rituals: Youth Subcultures in Post-War Britain. Londres: Hutchinson.

HonigmanN, J. (1953). Theory of Ritual. Chapel Hill: University of North Carolina.

LEACH, E. R. (1971). «La ritualisation chez l'homme par rapport à son développement culturel et social». En Le Comportement Rituel chez l'Homme et l'Animal, J. Huxley (ed.), 241-248. París: Gallimard.

Lotman, I. (1990). Universe of the Mind. A Semiotic Theory of Culture. Bloomington: Indiana University Press.

Miceli, S. (1972). Rito: la Forma e il Potere. Uomo $\alpha$ Cultura, Anno V N ${ }^{\circ}$ 10.

Scarduelli, P. (1988). Dioses, Espíritus, Ancestros. México: FCE.

STAAL, F. (1982). The science of Ritual. Poona (India): Bhandarkar Oriental Research Institute.

Thomas, L.-V. (1978). Prefacio a J.-D. Urbain. La Société de Conservation. Étude sémiologique des cimetières d'Occident. París: Payot. - (1983). Antropología de la Muerte. México: FCE.

Turner, V. (1969). The Ritual Process. Ithaca: Cornell University Press.

Urbain, J.-D. (1978). La Société de Conservation. Étude sémiologique des cimetières d'Occident. París: Payot.

Villa Posse, E. (1993). Muerte, Cultos y Cementerios. Bogotá: Disloque Editores.

Voller, J.G. (1991). «The Textuality of Death: Notes on the Reading of Cemeteries». Journal of American Culture 14:4, 1-9.

Vovelle, M. y Bertrand, R. (1983). La Ville des Morts. París. CNRS. 